\title{
Erratum to: Intermittent Obstructive Jaundice Following Gastric Band Placement
}

\author{
Nikolaos Arkadopoulos ${ }^{1} \cdot$ Christina Palamarou $^{2}$ - Andreas Koureas ${ }^{3}$. \\ Panagiotis Sechopoulos $^{2}$ - Athanassios Marinis ${ }^{1}$ - Konstantinos Kostopanagiotou ${ }^{1}$. \\ Nikolaos Dafnios ${ }^{1}$ - Vassilios Smyrniotis ${ }^{1}$
}

Published online: 16 February 2016

(C) Springer Science+Business Media New York 2016

Erratum to: OBSU 17(2): 247-250

DOI 10.1007/s11695-007-9029-y

The name of author Konstantinos Kostopanagiotou was spelled incorrectly in the original article.

The online version of the original article can be found at http://dx.doi.org/ 10.1007/s11695-007-9029-y.

\footnotetext{
Nikolaos Arkadopoulos narkado@otenet.gr

1 2nd Department of Surgery, University of Athens School of Medicine, Aretaieion Hospital, 21 Leonidiou Str., Kifissia, Athens, Greece 14564

2 "Henry Dunant" Hospital, Athens, Greece

3 1st Department of Radiology, University of Athens School of Medicine, Aretaieion Hospital, Athens, Greece
} 